\title{
Managing foot infections in patients with diabetes
}

\author{
Kerry Bowen, Diabetes Education Centre, Royal Newcastle Hospital, Newcastle, New \\ South Wales
}

\section{Summary}

Foot infections are a significant cause of morbidity for patients with diabetes and if left untreated can lead to amputation. Patients need to be instructed to wash, dry and examine their feet daily and are encouraged to seek medical attention promptly if they see signs of foot infection or new ulcer formation. Empirical use of antibiotics will often be necessary while awaiting the results of bacteriological and imaging investigations. When in doubt about the severity of infection urgent referral to a surgeon or specialist foot service for a second opinion is advised. Hospitalisation for observation, parenteral antibiotic therapy and possible surgical intervention may also be necessary. Diabetic arthropathy needs to be considered when signs mimicking infection are present in the absence of ulceration. Osteomyelitis or plantar space infection should be excluded as complicating factors if there is not rapid clinical improvement after starting antibiotic therapy.

Key words: antibiotics, ulceration.

(Aust Prescr 2007;30:21-4)

\section{Introduction}

Many people with long-standing diabetes mellitus are predisposed to foot injury, ulceration and infection because they have poor glycaemic control, peripheral vascular disease and/or peripheral neuropathy. For these patients even a trivial foot injury may rapidly lead to ulceration and infection. Everyone with diabetes should be advised to wash, dry and examine their feet daily and avoid excessive heat and cold, as well as trauma. Patients should seek medical attention promptly if they see signs of foot infection or new ulcer formation such as broken skin, changes in skin colour, bruising or swelling. If left untreated, diabetic ulcers can lead to amputation.

\section{How ulceration occurs}

When peripheral neuropathy predominates, the intrinsic muscles of the foot may waste, with clawing of the phalanges a result. This can lead to malposition of the fat pads that protect the plantar surfaces of the metatarsophalangeal joints causing adverse shearing and direct pressure on the tissue between the plantar surface and the now unprotected bony joints. These forces generate a reactive tissue oedema which breaks through to the skin surface to form an ulcer. When neuropathy is present, these ulcers may be painless. They may sometimes be quite large by the time they are detected if the patient is not carrying out daily foot inspection. Many of these shear-induced lesions will become infected, some even before surface ulceration presents itself. The presence of callus is often a marker for future ulceration. Callus should be pared back regularly by a suitablytrained podiatrist, nurse or medical practitioner. ${ }^{1}$

When ischaemia predominates, ulceration may often be initiated by ill-fitting footwear. Interdigital vessel occlusion may lead to ulceration and gangrene of a single digit. Obstruction more proximally may lead to wider gangrenous changes in the forefoot. On occasion this may be complicated by secondary bacterial infection.

\section{Microbiology}

In diabetes, infections that threaten the foot are usually caused by bacteria. Infected ulcers commonly have staphylococcal, streptococcal or facultative anaerobes such as Bacteroides species or faecal coliforms present. ${ }^{1,2}$ Single or multiple pathogens may be identified. Enteric pathogens can coexist with Streptococcus or Staphylococcus species in some instances. In humid climates fungal infections involving the web spaces of the foot may predispose an at-risk individual to secondary bacterial invasion.

\section{Assessment}

There are numerous algorithms and clinical pathways available to guide management of diabetic foot ulcers. ${ }^{2} \mathrm{~A}$ practical approach is to assess the ulcerated area for signs of active infection such as raised surface temperature, redness of the surrounding skin, necrosis, localised oedema and odour. Mapping of temperature changes over the foot can be carried out using an infra-red thermometer.

The presence of peripheral vascular disease can usually be determined quickly using observation and palpation. The capillary refill time is not considered to be sensitive or reliable enough to allow differentiation between vascular, ischaemic 
or neuroischaemic ulcers. Measurement of the ankle-brachial index or assessment of the arterial pressure waveforms of the posterior tibial and dorsalis pedis arteries using hand-held Doppler may provide further useful information. ${ }^{3}$

If infection is evident or suspected, deep wound swabs or needle aspiration of the exudate should be taken for bacteriological analysis before starting antibiotic treatment. If an ulcer can be probed down to bone then osteomyelitis is likely to be present. Surgical biopsy of the affected area may be useful for collection of subsequent specimens for microbiological examination if deterioration occurs after empirical antibiotic therapy is commenced.

It is usual to take a wound swab even if a patient is not exhibiting any clinical signs of infection in an ulcer which is clean, does not probe to bone, is not producing large amounts of exudate, and has granulation tissue. The swab should be taken as localised and as deep as possible. If a pathogen or commensal is present on a wound swab with no clinical signs of infection then a topical bacteriocidal dressing, such as one containing nanocrystalline silver, may help clear the wound of both types of bacteria.

A positive culture result may also help direct antibiotic treatment if overt infection subsequently develops.

The estimated depth and diameter of the wound should be recorded at each visit a tracing around the edge of the wound onto a sterile transparent double-layered plasticised dressing performed using a no-touch technique provides a useful record.

\section{Consult with a nuclear} medicine physician before a radioisotope scan is ordered as it may be of limited usefulness

surgeon should be able to make a decision on whether to explore and debride an ulcer based on clinical examination.

\section{Differential diagnoses}

Diabetic osteoarthropathy (Charcot's arthropathy) can often mimic a cellulitic process of the mid-foot or forefoot. Although a non-infective process in its pure form, it may sometimes present with sudden onset of oedema, redness, increased heat and sometimes pain. Elevation of the foot overnight can often help in making the diagnosis if X-ray signs are absent, as any oedema will often subside in the absence of infection. However, radionucleotide scanning must be performed if diabetic arthropathy is suspected, as increased isotope uptake in affected joints may be an early finding with this condition. Again, it is best to consult the nuclear medicine physician beforehand to ensure that the appropriate isotopes are used. MRI can also be used if available, as it may detect the bone oedema that can accompany diabetic arthropathy. Early diagnosis is important as appropriate treatment will prevent progressive foot deformity and subsequent disability.

Gout needs to be considered as a differential diagnosis when ulceration is not present and the diabetic patient presents with a swollen, hot, red and painful toe. The possibility of a fracture must not be forgotten. In all such cases, $\mathrm{X}$-ray is mandatory as a baseline and follow-up examination because in patients with diabetes, osteomyelitis may occasionally present as the

Additional tests that need to be performed on diabetic patients with foot infections include full blood count, erythrocyte sedimentation rate, electrolytes, $\mathrm{HbA} 1 \mathrm{c}$, plus renal and liver function tests. Weekly measurement of the C-reactive protein titre during treatment of a foot infection may help determine progress.

\section{Imaging}

When clinical signs of inflammation are evident, lateral, antero-posterior and oblique $X$-ray views of both feet should be performed with the $X$-ray request specifying the anatomical location of the ulcer and mentioning the possibility of underlying osteomyelitis, diabetic arthropathy and gas formation. Bone infection usually has to be present for several weeks before it is detectable on plain X-ray films, so serial $X$-rays at one to four weekly intervals may be necessary if clinical infection fails to resolve and the initial X-ray was clear.

If infection of bone or soft tissues is suspected it is prudent to consult with a nuclear medicine physician before a radioisotope scan is ordered as it may be of limited usefulness. Magnetic resonance imaging (MRI) of the affected area may be useful for differentiating infectious from non-infectious inflammatory conditions. ${ }^{2}$ However, if MRI facilities are not available a so-called 'sausage toe' - a hot and swollen toe - with or without accompanying ulceration of the phalanx.

Ischaemic foot ulcers may be painful and sometimes the surrounding tissues may appear erythematous. A thorough clinical examination with a positive Buerger's test suggests that ischaemia, not infection, is likely to predominate. When in doubt, antibiotics should be used empirically and the patient brought back within two or three days for review of progress.

\section{Antimicrobial therapy (see Fig. 1)}

The initial choice of outpatient-administered antibiotic therapy will be empirical. ${ }^{1}$ Where minimal inflammation is evident and the ulcer is both shallow and odourless a suitable wound dressing should suffice. If the ulcer is also malodorous an oral antibiotic can be trialled. Amoxycillin with clavulanic acid is a reasonable first choice. Dicloxacillin or flucloxacillin should be used when the clinical findings of localised erythema, swelling and heat without significant accompanying odour suggest that staphylococcal or streptococcal infection is likely. Clindamycin can be used in place of a penicillin if the patient has a history of penicillin hypersensitivity. Metronidazole combined with either dicloxacillin or flucloxacillin provides a reasonable oral antibiotic combination to use in systemically well patients, where an 
Fig. 1

Initial treatment guide for localised diabetic foot ulcers

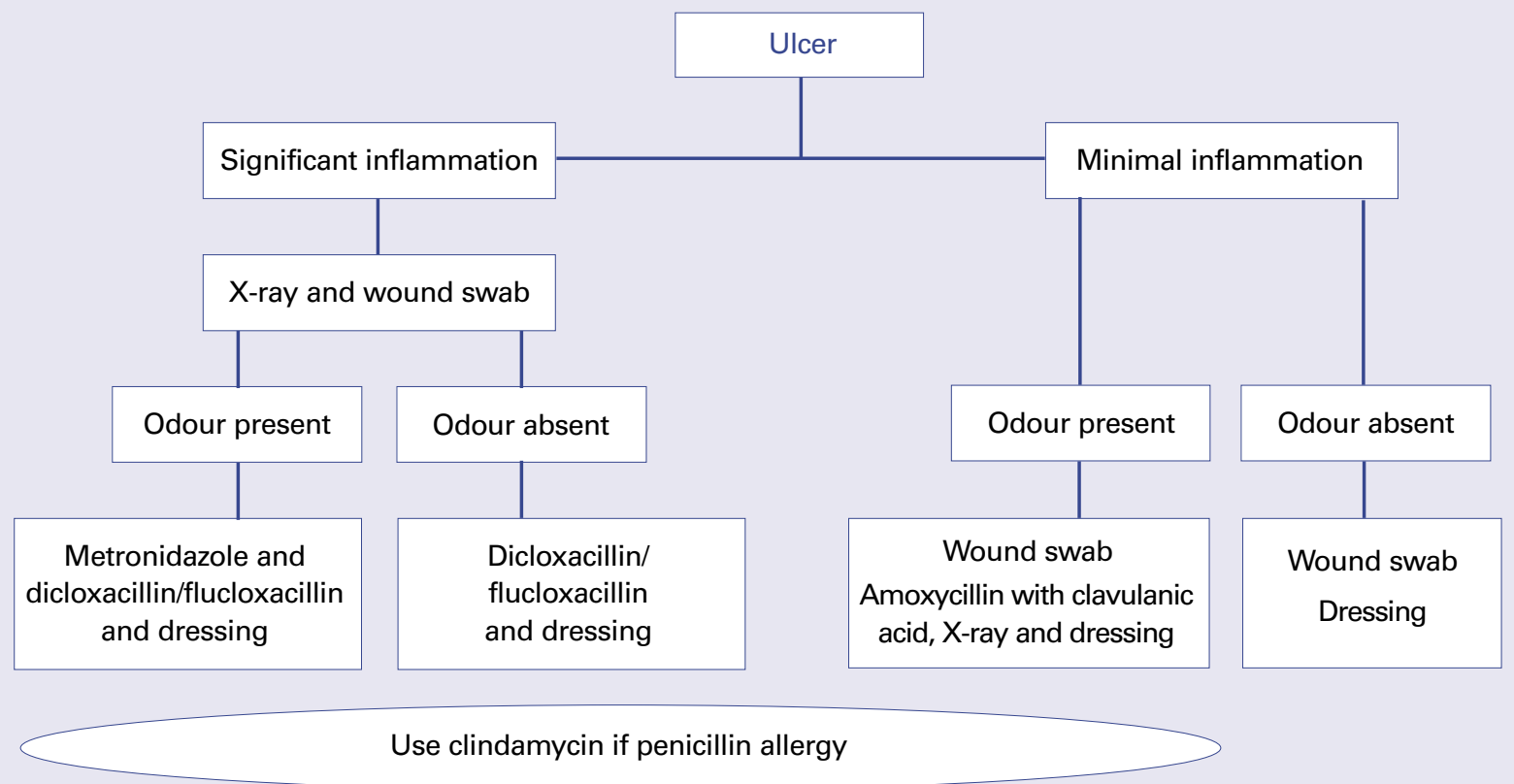

If phalangeal ulcer probes to bone, assume osteomyelitis and consider ciprofloxacin treatment for up to 8 weeks

If neuropathic ulcer over weight-bearing area is not healing, consider use of full contact cast

inflamed wound or ulcer appears localised with no necrosis but is malodorous, implying that the infection may be caused by a faecal organism or Bacteroides species. Occasionally, superficial pseudomonas infection is present, sometimes evident as a greenish hue over the surface of the ulcer. Application of a dilute acetic acid solution will often destroy this.

Antibiotic treatment should be given for at least 7-14 days in infections that appear to be localised to a digit. In more severe cases other indicators, such as a drop in C-reactive protein from significantly elevated to near normal, or arrest of bone destruction as shown on sequential $\mathrm{X}$-rays, may assist in determining the duration of antibiotic therapy.

If a phalangeal ulcer probes to bone, or if the diagnosis of osteomyelitis of the phalanx is made using radioisotope or $X$-ray evidence then resolution may sometimes be obtained with oral ciprofloxacin $500 \mathrm{mg}$ twice a day for 6-8 weeks. Removal of any loose sequestrum readily accessible to sterile forceps may hasten wound closure.

Vancomycin and rifampicin are commonly used if there is bacteriological evidence of methicillin-resistant Staphylococcus aureus (MRSA) infection in the wound and active involvement of the deep tissues is suspected. ${ }^{2}$ For MRSA infections it is helpful to seek the advice of a microbiologist or infectious diseases physician before commencing treatment.

Interdigital fungal foot infections may generate a portal of entry for bacteria and so foot hygiene is important. Methylated spirits can be used to keep the interdigital spaces dry and free of fungal colonisation.

\section{Dressings: broad principles}

If the ulcer is dry use a moisturising dressing such as a gel and avoid thin film dressings. If the wound is moist use an absorptive dressing such as foam or alginate. If the ulcer is superficially infected use a dressing that incorporates a bacteriocidal agent such as nanocrystalline silver.

\section{Other advice and follow-up}

Regular outpatient review will be required several times in the first week or two of antibiotic treatment if there is any doubt in the clinician's mind about the severity of the infection or if there is concern that the patient may not be able to adhere to the recommended treatment regimen. Patients must not put their weight on ulcers in weight-bearing areas. The application of a total contact cast from forefoot to below the knee, which is reapplied every 1-2 weeks, is the best way to achieve total 'off-loading' when all else has failed. Avoid a cast if the patient has significant peripheral vascular disease..$^{1,3}$ 
If pre-existing peripheral vascular disease is likely to hinder the healing process a vascular surgeon should assess the patient's suitability for a bypass or stenting procedure.

\section{Conclusion}

All foot infections in the diabetic patient need to be taken seriously. Small surface lesions may conceal significant deeper pathology requiring surgical intervention or aggressive antibiotic therapy. When in doubt about the severity of an infection, or if diabetic (Charcot's) arthropathy is suspected, seek an immediate second opinion from an orthopaedic surgeon or diabetes foot service. If this is not available then the patient should be admitted to hospital for observation and further investigations.

\section{References}

1. Jeffcoate WJ, Harding KG. Diabetic foot ulcers. Lancet 2003;361:1545-51. http://image.thelancet.com/extras/02art6190web.pdf [cited 2007 Jan 15]

2. Lipsky BA, Berendt AR, Deery HG, Embil JM, Joseph WS, Karchmer AW, et al. Diagnosis and treatment of diabetic foot infections. Clin Infect Dis 2004;39:885-910.

http://www.journals.uchicago.edu/CID/journal/issues/ v39n7/34365/34365.htm] [cited 2007 Jan 15]

3. Edmonds ME, Foster AV. Diabetic foot ulcers. BMJ 2006;332:407-10.

\section{Further reading}

Campbell LV, Graham AR, Kidd RM, Molloy HF, O'Rourke SR, Colagiuri S. The lower limb in people with diabetes. Position statement of the Australian Diabetes Society. Med J Aust 2000;173:369-72.

http://www.mja.com.au/public/issues/173_07_021000/campbell/ campbell.html [cited 2007 Jan 15]

National Institute for Health and Clinical Excellence. Type 2 diabetes - footcare: Algorithm.

http://www.nice.org.uk/page.aspx?o=208094 [cited 2007 Jan 15] http://www.diabetic-foot.com.au [cited 2007 Jan 15]

http://www.woundupdate.com [cited 2007 Jan 17]

http://www.emedicine.com/orthoped/topic387.htm [cited 2007 Jan 15]

Conflict of interest: none declared

\section{Self-test questions}

The following statements are either true or false (answers on page 27)

5. Infected diabetic ulcers may be painless.

6. Antibiotic treatment of a diabetic ulcer should not be started until the infecting organism is known.

\section{Your questions to the PBAC}

\section{Taxanes}

The listing of trastuzumab on the Pharmaceutical Benefits Scheme (PBS) in October 2006 was heralded with much fanfare. Along with this listing, changes to the prescribing requirements for taxanes also occurred. Both docetaxel and paclitaxel are now available on authority prescription for the treatment of HER2 positive early breast cancer in combination with trastuzumab. However, one group of patients will miss out on subsidised treatment. They are women with HER2 positive metastatic breast cancer who have not previously been treated with chemotherapy.

Patients with HER2 positive metastatic breast cancer can access trastuzumab under the Herceptin Access Program run through Medicare Australia. The prescribing restrictions for this program specify that the trastuzumab is to be used as a single drug or in combination with a taxane. Herein lies the problem. The current listing for taxanes on the PBS is 'advanced breast cancer after failure of prior therapy, which includes an anthracycline'. Patients with HER2 positive metastatic breast cancer who are chemotherapy naive cannot have the optimal therapy of trastuzumab in combination with a taxane, as the latter is not funded by the PBS.

Why were the taxanes made available for HER2 positive early breast cancer and not simply for all patients with HER2 positive breast cancer?

Jim Siderov

Senior Pharmacist

Cancer Services

Austin Health

Melbourne

\section{PBAC response:}

The Pharmaceutical Benefits Advisory Committee (PBAC) made its recommendation to subsidise taxanes for the treatment of HER2 positive early breast cancer in combination with trastuzumab because of evidence that this treatment combination met the requirements for PBS listing. The PBAC also recommended that the taxanes, in combination with an anthracycline and cyclophosphamide, be made available for adjuvant treatment for all patients with node positive breast 